\title{
One-Dimensional Cutting Stock Problem with Cartesian Coordinate Points
}

\author{
Niluka Rodrigo*, Sium Shashikala \\ Department of Mathematics, University of Peradeniya, Peradeniya, Sri Lanka \\ Email address: \\ nilukarodrigo@yahoo.co.uk (N. Rodrigo),sium92b@gmail.com (S. Shashikala) \\ ${ }^{*}$ Corresponding author
}

\section{To cite this article:}

Niluka Rodrigo, Sium Shashikala. One-Dimensional Cutting Stock Problem with Cartesian Coordinate Points. International Journal of Systems Science and Applied Mathematics. Vol. 2, No. 5, 2017, pp. 99-104. doi: 10.11648/j.ijssam.20170205.14

Received: September 29, 2016; Accepted: December 22, 2016; Published: October 24, 2017

\begin{abstract}
The cutting stock problem is used in many industrial processes and recently has been considered as one of the most important research topics. It is basically describes in two ways, One -dimensional and Two-dimensional Cutting Stock Problems (CSP). An optimum cutting stock problem can be defined as cutting a main sheet into smaller pieces while minimizing the total wastage of the raw material or maximizing overall profit obtained by cutting smaller pieces from the main sheet. In this study, One-dimensional CSP is discussed. Modified Brach and Bound algorithm for One-dimensional cutting stock problem is coded and programmed in the Matlab programming environment to generate feasible cutting patterns. At the same time, Cartesian coordinate points are derived from the developed algorithm. In our approach, the case study is pertained to the real data used at L.H. Chandrasekara \& Brothers (Pvt Ltd) in Sri Lanka for its production.
\end{abstract}

Keywords: One-Dimensional CSP, Brach and Bound Algorithm, Matlab Software, Cartesian Coordinate Points

\section{Introduction}

Efficiency of the production process has become a key factor of the success in today's competitive manufacturing environment in industries. Productivity can be improved by minimizing wastage, lead time and hence reducing the cost of production. Throughout the process of production, wastage can occur in many ways at any stage of the production process. Cutting stock problem can be defined as optimizing the raw material wastage. Therefore, techniques in Operations Research play a major role in minimizing wastage of raw material or maximizing usage of the raw material. In early days, in order to achieve the goal of (1DCSP), researchers used different methodologies. The linear programming based procedure was the first approach which may be traced back to the column generation procedure proposed by Gilmore and Gomary $(1961,1963)$. Moreover, Sequence Heuristic Procedure (SHP) described by Haesseler (1971) and then Hybrid Solution Procedure which is a combination of the LP based procedure and SHP or some other procedures (Haesseler et al., 1991). Basically CSP is describes in two ways, One-dimensional and Two- dimensional CSPs and it can be defined as cutting a raw material which is available in stock into smaller required items, which are known in size while minimizing the total wastage of the raw material or maximizing the overall profit obtained by cutting smaller items from the main sheet. Many researchers have worked on the CSP and developed different algorithms to solve the problem. Among them, the very first formulation of the CSP was formulated by Kantorovinch in 1939, although it was published 1960 (Kantorovinch, 1960). Gilmore et al [1] has proposed the linear programming approach, which solves the one-dimensional cutting stock problem. Unlimited number of raw materials with different lengths are available in stock is a one of assumptions of this study. A mathematical model has been developed to minimize the total cost of the raw material. Column Generation Algorithm has been developed to generate feasible cutting patterns. As a result of increasing the number of cutting items, the number of cutting patterns also increases. Linear programming problems which arise from cutting stock problems become intractable because of the large number of variables are involved (i.e. Linear programming technique is not applicable to solve mathematical model with too many variables). Gilmore [2] 
has made an approach for one-dimensional cutting stock problem as an extended of early study and cutting stock problem has been described as a NP-hard problem ${ }^{1}$. Gilmore [2] has described a new and faster Knapsack method, and mathematical formulation changes. The following formulation changes have been discussed; limitation on the number of cutting knives available, balancing of multiple machine usage when orders are being filled from more than one machine, and introduce a tolerance in the customers' orders. Gilmore has explained the procedure of Knapsack Method using a test problem ${ }^{2}$.

Addition to that, Morbito [3] has reported the problem of cutting rectangular plates into smaller ones in Brazilian hardboard industry. The problem was determine the best patterns to be cut by an automated machine composed of a set of circular saws, device to move and hold the plates and loading and unloading stations. A particular two-phase column generation procedure was described for the cutting stock formulation of hardboard industry. Each phase of the procedure was modeled as an integer program and solved by two alternative methods. The first was based on dynamic programming and the second was a simple extension of the algorithm presented in Gilmore [2]. Also, Saad M. A. Suliman [4] has developed a simple pattern generating procedure for solving the auxiliary problem which solves the one-dimensional CSP to minimize the trim loss or minimize the total amount of stock material. Branch and Bound Algorithm has been modified to find feasible cutting patterns for one-dimensional CSP and a search tree is used to develop the pattern generation method. Modified Branch and Bound Algorithm is illustrated using a case study, where standard steel coil which is $130 \mathrm{~cm}$ in length is cut into four different lengths of coils with similar width of main coil ${ }^{4}$. The onedimensional Cutting Stock Problem becomes more difficult when there are a large number of objects of different sizes available. A similar problem is addressed by Belov [5], and a method based on combination of some enumerative methods is proposed. In this work the more general case was studied where different stock types with supply bounds are available. The total material cost minimization has been modeled as an integer linear optimization problem ${ }^{5}$. Puchinger [6] has described a combined genetic algorithm/branch \& bound approach for solving a real world glass-cutting problem. The Genetic Algorithm uses an order-based representation, which is decoded using a greedy heuristic. The Branch \& Bound algorithm was applied with a certain probability enhancing the decoding phase by generating locally optimal subpatterns. Reported results indicate that the approach of occasionally solving sub patterns to optimality may increase the overall solution quality ${ }^{6}$. Fernandez [7] has presented a method to find Integer Solutions to One-dimensional CSP to minimize the trim loss or to minimize the number of master rolls needed to satisfy the orders. Two Integer linear programming models for the One-dimension CSP with different objective cost functions have been considered. Fernandez has studied an approach based on the classical column-generation procedure by Gilmore and Gomary for solving the linear programming (LP) relaxations. Also to obtain an integer solution, a final integer CSP after using an extra column-generation procedure has been solved ${ }^{7}$.

In addition to above studies, Rodrigo [8] has presented modified Branch and Bound Algorithm and a computer program using Matlab software package to generate feasible cutting patterns. As a case study, $1200 \mathrm{~cm}$ long steel bars are considered to be cut into eight types (different lengths) of pieces according to the customer requirements. Optimum cutting schedule has been presented by solving the integer linear programming problem which is given in the paper ${ }^{8}$. Rodrigo [9] has proposed a method to discover the feasible cutting patterns for One-Dimensional Cutting Stock Problem with varying dimensions. Pattern Generation Technique has been used to nest the pieces of cutting items within the main sheet by minimizing the raw material wastage. Computer program using Matlab software package has presented to generate feasible patterns. In the case study, three different paper rolls of widths $1000 \mathrm{~mm}, 500 \mathrm{~mm}$ and $800 \mathrm{~mm}$ are used as raw materials to cut different sizes of paper items according to the given specifications ${ }^{9}$. In addition to that Rodigo [10] has developed an algorithm based on Modified Branch And Bound to generate the cutting patterns for two-dimensional triangular shaped cutting items. As a case study, four different sizes of triangular shaped items with their demands are selected to cut from a raw material with known dimensions ${ }^{10}$.

\section{Materials and Methods}

\subsection{One-Dimensional (1D) Cutting Stock Problem}

In most industries, the cost of the materials consists of a high percent of total price (More than $80 \%$ ). The CSP is one of the most famous operation research problems which are defined to improve the quality of using materials. This CSP can be described as follow; there is a collection of rectangular shaped stock materials with different widths (but with the same length L) that we are going to divide them into smaller pieces of desired widths (with the same length L). First, the desired widths has been arranged in descending order $\left(w_{1}, w_{2}, \ldots ..\right)$ and then divide the original sheets into small pieces having the width- $w_{1}$ as much as possible. After then if there is a loss raw material, we go to the next required width- $w_{2}$ and divide that loss into small pieces having the width- $w_{2}$. By continuing the same procedure up to the smallest required width we can generate all the cutting patterns and finally calculate the minimum trim loss.

\subsection{Mathematical Model (Gilmore and Gomary, 1961)}

Any firm's main objective is to maximize the annual contribution margin accruing from its production and sales. By reducing wastages and maximizing sales, productivity can be improved. Wastage can occur in many ways and at any step of production line cutting stock problem can be described under the raw material wastage. According to the selection, a mathematical model to minimize the raw material wastage is formulated as follows: 


$$
\begin{gathered}
\text { Minimize } z=\sum_{k=1}^{\mathrm{h}} \sum_{j=1}^{m} c_{k j} x_{k j} \text { (Total Cutloss) } \\
\text { Subject to } \sum_{k=1}^{\mathrm{h}} \sum_{j=1}^{\mathrm{m}} a_{i k j} x_{k j} \geq d_{i} \\
\text { for all } i=1,2, \ldots, n \quad \text { (Demand Constraints) } \\
\qquad x_{k j}, a_{i k j} \geq 0 \text { and integer for all } i, j, k
\end{gathered}
$$

Where

$h=$ Number of raw materials with different sizes,

$n=$ Number of items,

$m=$ Number of patterns,

$a_{i k j}=$ The number of occurrences of the $i^{\text {th }}$ item of the $k^{\text {th }}$ sheet in the $j^{\text {th }}$ pattern,

$c_{k j}=$ Cut loss of the $k^{\text {th }}$ sheet in the $j^{\text {th }}$ pattern,

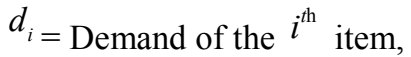

$x_{k j}=$ The number of main sheets being cut from the $k^{\text {th }}$ sheet according to the $j^{\text {th }}$ pattern.

\subsection{Modified Branch and Bound Algorithm (in Step-Wise Form)}

Step1: Arrange the required widths of raw materials, $W_{k}, k=1,2, \ldots$, hin decreasing order.

(i.e. $W_{1}>W_{2}>\cdots>W_{h}$ where $\mathrm{h}$ is the number of main sheets with different widths)

Step 2: Arrange the required widths, $w_{i}, i=1,2, \ldots, n$ in decreasing order.

(i.e. $w_{1}>w_{2}>\cdots>w_{n}$ where $\mathrm{n}$ is the number of items with different widths)

Step 3: For $i=1,2, \ldots, n ; k=1,2, \ldots, h$ and $j=1$ do Step 4 and Step5.

Step 4:Set $a_{1 k 1}=\left[\left[W_{k} / w_{1}\right]\right]$;

$$
a_{i k j}=\left[\left[\left(W_{k}-\sum_{\mathrm{z}=1}^{i-1} a_{z j k} w_{z}\right) / w_{i}\right]\right]
$$

Where $W_{k}$ is the length of the $k^{\text {th }}$ main sheet. Here, ${ }^{a_{i j k}}$ is the number of pieces of the $i^{\text {th }}$ item in the $j^{\text {th }}$ pattern along the width of the $k^{\text {th }}$ main sheet and $[[y]]$ is the greatest integer less than or equal to $\mathrm{y}$.

$$
\text { Step } 5: c_{k j}=W_{k}-\sum_{i=1}^{n} a_{i k j} \cdot w_{i}
$$

find the cut loss resulting from cut pattern 1.

Step 6: Set the level index $i$ to $n$-1 (i.e. set $t=n-1$ )

While $\mathrm{t}>0$ do Step7.

Step 7: While $a_{t k j}>0$
Set $j=j+1$ and do Step8.

Step 8: Generate a new pattern according to the following conditions;

$$
\begin{aligned}
& \text { For } z=1,2, \ldots, t-1 \\
& \text { Set } a_{z k j}=a_{z k(j-1)} \\
& \text { For } z=t \\
& \text { Set } a_{z k j}=a_{z k(j-1)}-1 \text {; } \\
& \text { For } z=t+1, \ldots, n \\
& \text { Calculate } a_{z k j} \text { using }(1) \text {. } \\
& \text { Step 9:Set } t=t-1 \\
& \text { Step 10: Stop. }
\end{aligned}
$$

\subsection{Case Study}

Modified branch and bound algorithm is tested to determine feasible and optimal cutting patterns.

A paper company (Chandrasekara Group Of Companies in Sri Lanka) uses Bank papers of widths $80 \mathrm{~cm}$ and $60 \mathrm{~cm}$ with same length $(90 \mathrm{~cm})$ as raw materials to cut different sizes paper items according to the given specifications. The company has received an order for papers with the following specifications:

Table 1. Required cutting item dimensions and demands.

\begin{tabular}{llllll}
\hline Order Number (i) & $\mathbf{1}$ & $\mathbf{2}$ & $\mathbf{3}$ & $\mathbf{4}$ & $\mathbf{5}$ \\
\hline Name of the Item & A2 & B4 & A4 & B5 & Legal \\
Required width $w_{i}(\mathrm{~cm})$ & 42 & 26 & 21 & 18 & 8.5 \\
Demand & 1000 & 2000 & 4000 & 5000 & 6000 \\
\hline
\end{tabular}

Bank Papers are produced by the company in two standard widths; $60 \mathrm{~cm}$ and $80 \mathrm{~cm}$ that are slit to the sizes specified by the orders.

\section{Results and Discussion}

Modified Branch and Bound Algorithm is used to the above example to generate feasible cutting patterns as given below;

Step 1: For $k=1,2$ widths of rawmaterials $W_{k}=80 \mathrm{~cm}$ and $60 \mathrm{~cm}$.

Step 2: For $i=1,2,3,4,5$ widths of items $w_{i}=42 \mathrm{~cm}$, $26 \mathrm{~cm}, 21 \mathrm{~cm}, 18 \mathrm{~cm}, 8.5 \mathrm{~cm}$.

Step 3: For $i=1,2, \ldots, 5$ and $k=1,2$ and $j=1$, do Step 4 and Step 5.

Step 4: Allocating cutting items for cutting pattern 1, Item 1 is selected and nested along the width of the raw material.

Following equation states the maximum number of pieces that can be allocated for Item 1 .

$$
a_{111}=\left[\left[W_{1} / w_{1}\right]\right]
$$

Residual width of the raw material calculated by the formula bellow:

$$
\begin{aligned}
& \text { Residual width }=W_{1}-\left(w_{1} a_{111}\right) . \\
& \text { If } w_{2} \leq W_{1}-\left(w_{1} a_{111}\right),
\end{aligned}
$$

where $w_{2}$ is the width of the Item 2 . Then Item 2 is nested within the residual width of the raw material. 
Nesting of Item 2 is as follows:

$$
a_{121}=\left[\left[\left[W_{1}-\left(w_{1} a_{111}\right)\right] / w_{2}\right]\right]
$$

This process continues till the last cutting item (Item 5) while calculating residual width of the raw material.

$$
\begin{gathered}
\text { Set } a_{111}=\left[\left[W_{1} / w_{1}\right]\right]=1 ; \\
a_{211}=\left[\left[\left[W_{1}-\left(w_{1} a_{111}\right)\right] / w_{2}\right]\right]=1 ; \\
a_{311}=\left[\left[\left[W_{1}-\left(w_{1} a_{111}\right)-\left(w_{2} a_{211}\right)\right] / w_{3}\right]\right]=0 ; \\
a_{411}=\left[\left[\left[W_{1}-\left(w_{1} a_{111}\right)-\left(w_{2} a_{211}\right)-\left(w_{3} a_{311}\right)\right] / w_{4}\right]\right]=0 ; \\
a_{511}=\left[\left[\left[W_{1}-\left(w_{1} a_{111}\right)-\left(w_{2} a_{211}\right)-\left(w_{3} a_{311}\right)-\left(w_{4} a_{411}\right)\right] / w_{5}\right]\right]=1 .
\end{gathered}
$$

Nested cutting items in Step 2 is defined as first cutting pattern

Set pattern $1=\left[\begin{array}{l}1 \\ 1 \\ 0 \\ 0 \\ 1\end{array}\right]$

Step 5: Total cut loss

Cutloss is calculated as follows:

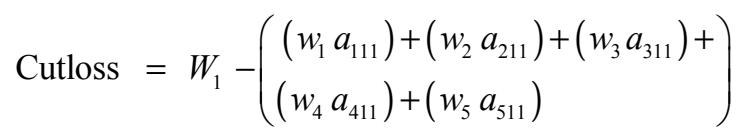

$$
=3.5 \mathrm{~cm}
$$

Step 6: At the end of each pattern,

Set $i=n-1(>0)$.

Set $i=4$.

Step 7: Value of $a_{i k j}$ is checked. If $a_{i k j}=0$, setting $i=i-1$ and again value of $a_{i k j}$ is checked. When $a_{i k j}>0$, set $j=j+1$ and determine the number of pieces of each cutting item along the length of the raw material.

$$
\begin{gathered}
a_{411}=0, \quad \text { set } j=j . \\
a_{311}=0 \\
a_{211}>0
\end{gathered}
$$

Set $j=j+1$

Step 8: Generate a new pattern according to the following conditions:

\begin{tabular}{|c|c|c|c|c|c|c|c|c|c|c|c|c|c|c|c|}
\hline \multirow{2}{*}{$\begin{array}{l}\text { Required } \\
\text { widths }\end{array}$} & \multicolumn{15}{|c|}{ Cutting patterns(from $80 \mathrm{~cm}$ raw material) } \\
\hline & 1 & 2 & 3 & 4 & 5 & 6 & 7 & 8 & 9 & 10 & 11 & 12 & 13 & 14 & 15 \\
\hline $42 \mathrm{~cm}$ & 1 & 1 & 1 & 1 & 0 & 0 & 0 & 0 & 0 & 0 & 0 & 0 & 0 & 0 & 0 \\
\hline $26 \mathrm{~cm}$ & 1 & 0 & 0 & 0 & 0 & 3 & 2 & 2 & 2 & 1 & 1 & 1 & 1 & 1 & 1 \\
\hline $21 \mathrm{~cm}$ & 0 & 1 & 0 & 0 & 0 & 0 & 1 & 0 & 0 & 2 & 1 & 1 & 0 & 0 & 0 \\
\hline $18 \mathrm{~cm}$ & 0 & 0 & 2 & 1 & 0 & 0 & 0 & 1 & 0 & 0 & 1 & 0 & 3 & 2 & 1 \\
\hline $8.5 \mathrm{~cm}$ & 1 & 2 & 0 & 2 & 4 & 0 & 0 & 1 & 3 & 1 & 1 & 3 & 0 & 2 & 4 \\
\hline Cut loss $(\mathrm{cm})$ & 3.5 & 0 & 2 & 3 & 4 & 2 & 7 & 1.5 & 2.5 & 3.5 & 6.5 & 7.5 & 0 & 1 & 2 \\
\hline Poruimd uidtho & \multicolumn{15}{|c|}{ Cutting patterns(from $80 \mathrm{~cm}$ raw material) } \\
\hline $42 \mathrm{~cm}$ & 0 & 0 & 0 & 0 & 0 & 0 & 0 & 0 & 0 & 0 & 0 & 0 & 0 & 0 & \\
\hline $26 \mathrm{~cm}$ & 1 & 0 & 0 & 0 & 0 & 0 & 0 & 0 & 0 & 0 & 0 & 0 & 0 & 0 & \\
\hline $21 \mathrm{~cm}$ & 0 & 3 & 2 & 2 & 2 & 1 & 1 & 1 & 1 & 0 & 0 & 0 & 0 & 0 & \\
\hline $18 \mathrm{~cm}$ & 0 & 0 & 2 & 1 & 0 & 3 & 2 & 1 & 0 & 4 & 3 & 2 & 1 & 0 & \\
\hline $8.5 \mathrm{~cm}$ & 6 & 2 & 0 & 2 & 4 & 0 & 2 & 4 & 6 & 0 & 3 & 5 & 7 & 9 & \\
\hline Cut loss $(\mathrm{cm})$ & 3 & 0 & 2 & 3 & 4 & 5 & 6 & 7 & 8 & 8 & 0.5 & 1.5 & 2.5 & 3.5 & \\
\hline Reauived widths & \multicolumn{15}{|c|}{ Cutting patterns(from $60 \mathrm{~cm}$ raw material) } \\
\hline Required widths & 30 & 31 & 32 & 33 & 34 & 35 & 36 & 37 & 38 & 39 & 40 & 41 & 42 & 43 & 44 \\
\hline $26 \mathrm{~cm}$ & 0 & 0 & 2 & 1 & 1 & 1 & 0 & 0 & 0 & 0 & 0 & 0 & 0 & 0 & 0 \\
\hline $21 \mathrm{~cm}$ & 0 & 0 & 0 & 1 & 0 & 0 & 2 & 2 & 1 & 1 & 1 & 0 & 0 & 0 & 0 \\
\hline
\end{tabular}

$$
\begin{gathered}
\text { Set } a_{112}=a_{111}=1 ; \\
\text { Set } a_{212}=a_{211}-1=0 ; \\
a_{312}=\left[\left[\left[W_{1}-\left(w_{1} a_{112}\right)-\left(w_{2} a_{212}\right)\right] / w_{3}\right]\right]=1 ; \\
a_{412}=\left[\left[\left[W_{1}-\left(w_{1} a_{112}\right)-\left(w_{2} a_{212}\right)-\left(w_{3} a_{312}\right)\right] / w_{4}\right]\right]=0 ; \\
a_{512}=\left[\left[\left[w_{1}-\left(w_{1} a_{112}\right)-\left(w_{2} a_{212}\right)-\left(w_{3} a_{312}\right)-\left(w_{4} a_{412}\right)\right] / w_{5}\right]\right]=2 .
\end{gathered}
$$

Nested cutting items of pattern 2 as follows:

Set Pattern $2=\left[\begin{array}{l}1 \\ 0 \\ 1 \\ 0 \\ 2\end{array}\right]$.

Step 5: Total cut loss

$$
c_{12}=\left[W_{1}-\sum_{i=1}^{5} a_{i 12} w_{i}\right]=0 \mathrm{~cm}
$$

Repeat the procedure until $i=5(n)$.

This process ends up with 44 different cutting patterns to allocate 5 items which is exhibited in the Table 2 .

Table 2. Generated Cutting Patterns. 


\begin{tabular}{|c|c|c|c|c|c|c|c|c|c|c|c|c|c|c|c|}
\hline \multirow{2}{*}{$\begin{array}{l}\text { Required } \\
\text { widths }\end{array}$} & \multicolumn{15}{|c|}{ Cutting patterns(from $80 \mathrm{~cm}$ raw material) } \\
\hline & 1 & 2 & 3 & 4 & 5 & 6 & 7 & 8 & 9 & 10 & 11 & 12 & 13 & 14 & 15 \\
\hline $18 \mathrm{~cm}$ & 1 & 0 & 0 & 0 & 1 & 0 & 1 & 0 & 2 & 1 & 0 & 3 & 2 & 1 & 0 \\
\hline $8.5 \mathrm{~cm}$ & 0 & 2 & 0 & 1 & 1 & 4 & 0 & 2 & 0 & 2 & 4 & 0 & 2 & 4 & 7 \\
\hline
\end{tabular}

There are 44 feasible cutting patterns available to cut raw materials with the dimensions $80 \mathrm{~cm}$ and $60 \mathrm{~cm}$ into required items. By considering the standard lengths of the main sheets (length of the main sheets are same $(90 \mathrm{~cm})$ ) and the other items;

Table 3. Length-wise allocation.

\begin{tabular}{lll}
\hline Item & Standard length & No. of items* (cuts are made length-wise) \\
\hline A3 & $59.4 \mathrm{~cm}$ & 1 \\
B4 & $36.5 \mathrm{~cm}$ & 2 \\
A4 & $29.7 \mathrm{~cm}$ & 3 \\
B5 & $26 \mathrm{~cm}$ & 3 \\
Legal & $14 \mathrm{~cm}$ & 6 \\
\hline
\end{tabular}

(No. of items* - number of items that can be cut from the relevant strip after cutting the main sheet width-wise). Then the total number of cutting patterns can be listed as follows:

Table 4. Feasible cutting patterns.

\begin{tabular}{|c|c|c|c|c|c|c|c|c|c|c|c|c|c|c|c|}
\hline \multirow{2}{*}{$\begin{array}{l}\text { Required } \\
\text { widths }\end{array}$} & \multicolumn{15}{|c|}{ Cutting patterns(from $80 \times 90 \mathrm{~cm}^{2}$ rawmaterial) } \\
\hline & 1 & 2 & 3 & 4 & 5 & 6 & 7 & 8 & 9 & 10 & 11 & 12 & 13 & 14 & 15 \\
\hline $42 \mathrm{~cm}$ & 1 & 1 & 1 & 1 & 0 & 0 & 0 & 0 & 0 & 0 & 0 & 0 & 0 & 0 & 0 \\
\hline $26 \mathrm{~cm}$ & 2 & 0 & 0 & 0 & 0 & 6 & 4 & 4 & 4 & 2 & 2 & 2 & 2 & 2 & 2 \\
\hline $21 \mathrm{~cm}$ & 0 & 3 & 0 & 0 & 0 & 0 & 3 & 0 & 0 & 6 & 3 & 3 & 0 & 0 & 0 \\
\hline $18 \mathrm{~cm}$ & 0 & 0 & 6 & 3 & 0 & 0 & 0 & 3 & 0 & 0 & 3 & 0 & 9 & 6 & 3 \\
\hline $8.5 \mathrm{~cm}$ & 6 & 12 & 0 & 12 & 24 & 0 & 0 & 6 & 18 & 6 & 6 & 18 & 0 & 12 & 24 \\
\hline $\begin{array}{l}\text { Cut } \\
\operatorname{loss}\left(\mathrm{cm}^{2}\right)\end{array}$ & 2093.2 & 1406.1 & 1897.2 & 1873.2 & 1849.2 & 1506 & 1532.9 & 1286 & 1262 & 845.8 & 1312.9 & 1288.9 & 1090 & 1066 & 1042 \\
\hline Required & \multicolumn{15}{|c|}{ Cutting patterns(from $80 \times 90 \mathrm{~cm}^{2}$ rawmaterial) } \\
\hline widths & 16 & 17 & 18 & 19 & 20 & 21 & 22 & 23 & 24 & 25 & 26 & 27 & 28 & 29 & \\
\hline $42 \mathrm{~cm}$ & 0 & 0 & 0 & 0 & 0 & 0 & 0 & 0 & 0 & 0 & 0 & 0 & 0 & 0 & \\
\hline $26 \mathrm{~cm}$ & 2 & 0 & 0 & 0 & 0 & 0 & 0 & 0 & 0 & 0 & 0 & 0 & 0 & 0 & \\
\hline $21 \mathrm{~cm}$ & 0 & 9 & 6 & 6 & 6 & 3 & 3 & 3 & 3 & 0 & 0 & 0 & 0 & 0 & \\
\hline $18 \mathrm{~cm}$ & 0 & 0 & 6 & 3 & 0 & 9 & 6 & 3 & 0 & 12 & 9 & 6 & 3 & 0 & \\
\hline $8.5 \mathrm{~cm}$ & 36 & 12 & 0 & 12 & 24 & 0 & 12 & 24 & 36 & 0 & 18 & 30 & 42 & 54 & \\
\hline $\begin{array}{l}\text { Cut } \\
\operatorname{loss}\left(\mathrm{cm}^{2}\right)\end{array}$ & 1018 & 158.7 & 649.8 & 625.8 & 601.8 & 1116.9 & 1092.9 & 1068.9 & 1044.9 & 1584 & 846 & 822 & 798 & 774 & \\
\hline Required & \multicolumn{15}{|c|}{ Cutting patterns(from $60 \times 90 \mathrm{~cm}^{2}$ rawmaterial) } \\
\hline widths & 30 & 31 & 32 & 33 & 34 & 35 & 36 & 37 & 38 & 39 & 40 & 41 & 42 & 43 & 44 \\
\hline $42 \mathrm{~cm}$ & 1 & 1 & 0 & 0 & 0 & 0 & 0 & 0 & 0 & 0 & 0 & 0 & 0 & 0 & 0 \\
\hline $26 \mathrm{~cm}$ & 0 & 0 & 4 & 2 & 2 & 2 & 0 & 0 & 0 & 0 & 0 & 0 & 0 & 0 & 0 \\
\hline $21 \mathrm{~cm}$ & 0 & 0 & 0 & 3 & 0 & 0 & 6 & 6 & 3 & 3 & 3 & 0 & 0 & 0 & 0 \\
\hline $18 \mathrm{~cm}$ & 3 & 0 & 0 & 0 & 3 & 0 & 3 & 0 & 6 & 3 & 0 & 9 & 6 & 3 & 0 \\
\hline $8.5 \mathrm{~cm}$ & 0 & 12 & 0 & 6 & 6 & 24 & 0 & 12 & 0 & 12 & 24 & 0 & 12 & 24 & 42 \\
\hline $\begin{array}{l}\text { Cut } \\
\operatorname{loss}\left(\mathrm{cm}^{2}\right)\end{array}$ & 1501.2 & 1477.2 & 1604 & 916.9 & 1384 & 646 & 253.8 & 229.8 & 720.9 & 696.9 & 672.9 & 1188 & 1164 & 1140 & 402 \\
\hline
\end{tabular}

There are 44 feasible cutting patterns available to cut rawmaterials with the dimensions $80 \times 90 \mathrm{~cm}$ and $60 \times$ $90 \mathrm{~cm}$ into required items. The mathematical model is solved as an integer programming model using EXCEL to design generated cutting patterns so that the trim loss will be minimized and optimum solution to the model is given in Table 5.

Table 5. Optimum cutting schedule.

\begin{tabular}{llllll}
\hline \multirow{2}{*}{ Item } & \multicolumn{9}{l}{ Patterns } & \multirow{2}{*}{ Demand } \\
\cline { 2 - 5 } & $\mathbf{6}$ & $\mathbf{3 0}$ & $\mathbf{3 5}$ & $\mathbf{3 6}$ & \\
\hline A2 & 0 & 1 & 0 & 0 & 1000 \\
B4 & 6 & 0 & 2 & 0 & 2000 \\
A4 & 0 & 0 & 0 & 6 & 4000 \\
B5 & 0 & 3 & 0 & 3 & 5000 \\
Legal & 0 & 0 & 24 & 0 & 6000 \\
Cut loss $\left(\mathrm{cm}^{2}\right)$ & 1506 & 1501.2 & 646 & 253.8 & Total Cutloss \\
No. of $\operatorname{sheets}\left(\boldsymbol{x}_{\boldsymbol{k} \boldsymbol{j}}\right)$ & 250 & 1000 & 250 & 667 & 2208484.6 \\
\hline
\end{tabular}

\section{Conclusion}

In this study, a cutting stock problem is formulated as a mathematical model based on the concept of cutting patterns. As shown in the Table 2, forty four feasible cutting patterns were generated and after solving the model, only four patterns were selected as given in Table 5 to cut the main sheets according to the requirements. In this case study, the plant assumes that all extra pieces from each item were stocked for the next production. Since the company cuts only one item from one main sheet, they need minimum 500 main sheets from $80 \times 90 \mathrm{~cm}^{2}$ and 1642 main sheets from $80 \times$ $90 \mathrm{~cm}^{2}$ to satisfy the given demand. According to our process we need only 250 main sheets from $80 \times 90 \mathrm{~cm}^{2}$ and 1917 main sheets from $80 \times 90 \mathrm{~cm}^{2}$ to satisfy the demand. 
Table 6. Number of sheets required to satisfy the demand according to the company process.

\begin{tabular}{|c|c|c|c|c|c|c|}
\hline Item & Length (cm) & Width (cm) & No. of items (that can be cut from one sheet) & Demand & No. of sheets required & No. of the main sheet \\
\hline A2 & 59.4 & 42 & 2 & 1000 & 500 & 1 \\
\hline B4 & 36.5 & 26 & 6 & 2000 & 500 & 2 \\
\hline A4 & 29.7 & 21 & 8 & 4000 & 500 & 2 \\
\hline B5 & 26 & 18 & 10 & 5000 & 500 & 2 \\
\hline Legal & 14 & 8.5 & 42 & 6000 & 142 & 2 \\
\hline
\end{tabular}

The total cutloss of the company $=2525200 \mathrm{~cm}^{2}$

Rate of utilized area (of the company) $=79.77 \%$

Total cutloss (obtained from our model) $=2208484.6 \mathrm{~cm}^{2}$

Rate of utilized area $=81.83 \%$

(obtained from our model)

Clearly we can see that the optimum solution we obtained through our process is more profitable than the existing one.

\section{References}

[1] P. C. Gilmore and R. E. Gomary, "A Linear Programming Approach to the Cutting Stock Problem", Operations Research, Vol. 9, No. 2 (1961), 849-859.

[2] P. C. Gilmore and R. E. Gomary, "A Linear Programming Approach to the Cutting Stock Problem - Part II", Operations Research, Vol. 11, No. 6 (1963), 863-888.

[3] R. Morbito and V. Garcia, "A Cutting Stock Problemin Hardboard Industry- Case Study", Computer Operations Research, Vol. 25, No. 6 (1997), 469-485.

[4] Saad M. A. Suliman, "Pattern Generating Procedure for the Cutting Stock Problem", International Journal of Production Economics 74(2001) 293-301.

[5] G. Belov and G. Scheithauer, "A cutting plane algorithm for the one-dimensional cutting stock problem with multiple stock lengths", European Journal of Operational Research 141 (2002) 274-29.

[6] JakobPuchinger, Gunther R. Raidl and Gabriele Koller, "Solving a Real-World Glass Cutting Problem".

[7] L. Fern'andez, L. A. Fern'andez, C. Pola, "Integer Solutions to Cutting Stock Problems", $2^{\text {nd }}$ International Conference on Engineering Optimization, Sep 6-9, 2010.

[8] W. N. P Rodrigo, W. B Daundasekera and A. A. I Perera, "Pattern Generation for One-dimensional Cutting Stock Problem", Peradeniya University Research Session (PURSE), 2011.

[9] W. N. P. Rodrigo, W. B. Daundasekera and A. A. I. Perera, "Modified Method for One-dimensional Cutting Stock Problem", Science Publishing Group 2015;3(3); 12-17.

[10] W. N. P. Rodrigo, W. B. Daundasekera and A. A. I. Perera, “A Method for Two-Dimensional Cutting Stock Problem with Triangular Shape Items", British Journal of Mathematics and Computer Science 2013;3(4);750-771. 